\title{
IL-6-induced pathophysiology during pre-eclampsia: potential therapeutic role for magnesium sulfate?
}

This article was published in the following Dove Press journal:

International Journal of Interferon, Cytokine and Mediator Research

I 3 July 201 I

Number of times this article has been viewed

\section{Babbette LaMarca \\ Justin Brewer \\ Kedra Wallace}

Department of Obstetrics and Gynecology, University of Mississippi

Medical Center, Jackson, MS, USA
Correspondence: Babbette LaMarca

Departments of Obstetrics and

Gynecology and Physiology,

Division of Maternal Fetal Medicine,

University of Mississippi Medical Center,

2500 North State Street, Jackson,

MS 39216-4505, USA

$\mathrm{Tel}+\mathrm{I} 60|8| 5 \mid 430$

Fax +l 60I8I5 I446

Email bblamarca@umc.edu
Abstract: Pre-eclampsia is defined as new onset hypertension with proteinuria during pregnancy. Pre-eclampsia is also characterized by endothelial cell activation and dysfunction and intrauterine growth restriction. Preeclamptic women display a chronic inflammatory response characterized by elevated inflammatory cytokines, circulating monocytes, neutrophils, and T and B lymphocytes secreting autoantibodies that activate the angiotensin II type I receptor (AT1-AA). Although the pathophysiology of pre-eclampsia is becoming more defined, the genesis of the disease is still largely unknown. Furthermore, the only treatment for extreme forms of the disease is bed rest and administration of magnesium sulfate to sustain the pregnancy a few days prior to early delivery of the fetus, which can lead to devastating neurological and physical effects for the newborn. Administration of magnesium sulfate is routinely given without adverse effects. The focus of this review is to discuss the cascade of events leading to cytokines, specifically interleukin-6 (IL-6), in stimulating vasoactive substances such as AT1-AA (Figure 1) and to examine the mechanism whereby administration of magnesium sulfate can be beneficial during pre-eclampsia. One area is to decrease vascular resistance index parameters determined by Doppler velocimetry. Another potential area of benefit with magnesium sulfate administration may be to decrease inflammatory responses or decrease cardiovascular mechanisms stimulated by overexpression of inflammatory cytokines in response to placental ischemia or animal models of elevated IL-6 during pregnancy. Further studies identifying IL-6-driven mechanisms playing a role in the development of hypertension during pregnancy and how administration of magnesium sulfate can suppress them are critical to improve decisions affecting patient care in women with pre-eclampsia. The results of these types of studies will be advantageous to further our knowledge of the pathophysiological ramifications associated with pre-eclampsia and to further therapeutic development for this disease.

Keywords: inflammation, interleukin-6, pregnancy, hypertension

\section{Introduction}

Pre-eclampsia is a disorder that occurs in 4\%-8\% of pregnancies and is characterized by new-onset proteinuria and hypertension after 20 weeks gestation. Some patients will develop more severe consequences of pre-eclampsia such as renal failure, disseminated intravascular coagulation, pulmonary edema, HELLP (hemolysis, elevated liver enzymes, low platelet count) syndrome, and placental abruption. ${ }^{1-16}$ When seizures occur in the setting of pre-eclampsia, the disorder is referred to as eclampsia. This occurs in about $0.5 \%$ of patients with mild pre-eclampsia and up to $2 \%-3 \%$ of those with severe disease. ${ }^{1-16}$ Much obstetric research has been directed at avoiding these consequences, specifically the occurrence of eclamptic seizures and exacerbation of the disease into pulmonary edema and HELLP syndrome. 
Pre-eclampsia has long been considered a disease of immunological origin, characterized by elevated inflammatory cytokines, activation of various immune cells and production of autoantibodies that activate the angiotensin II type 1 receptor (AT1-AA). ${ }^{16-30}$ Many inflammatory cells are activated in the circulation and infiltrate into renal and placental tissues. Macrophages, neutrophils, and T lymphocytes of the Thelper (Th)-1 subset are the predominant cell types mediating the inflammatory cascade in preeclamptic women. ${ }^{16-30}$ Furthermore, the cytokine profile of women with pre-eclampsia is consistent with a cell-mediated immune response that utilizes neutrophils, macrophages, and a cluster of differentiation (CD)4+Th-1 cells as a defense mechanism against microbial infections. As a result, elevated inflammatory cytokines and the oxidative burst of phagocytic cells persist, resulting in vascular oxidative stress during pre-eclampsia. ${ }^{16-33}$ In addition, women with pre-eclampsia exhibit abnormal ratios of Foxp3(+) (forkhead box p3) Treg to interleukin (IL)-17-expressing CD4+ T cells. ${ }^{34}$ The percentage of CD4+IL-17-producing $\mathrm{T}$ cells is decreased significantly in normal pregnant women compared with those with evidence of pre-eclampsia. Moreover, IL-17 and IFN- $\gamma$ (interferon gamma) is increased while IL-10 is decreased with pre-eclampsia compared with those with normal gestation. AT1-AA has been shown to be produced by all women with reductions in placental blood flow examined thus far, and production of the autoantibody is not terminated upon delivery of the fetus and placenta. ${ }^{35,36}$ It is unknown how long or to what extent these women continue to produce AT1-AA or whether it plays a role in the development of cardiovascular disease, which is prevalent later in life for preeclamptic women. Defining the pathway of AT1-AA production will aid in understanding its reoccurrence in preeclamptic women. Chronic overexpression of the AT1-AA via activated T cell dependent mechanisms could contribute to a decline in renal function leading to increased blood pressure among preeclamptics.

Many investigators believe that pre-eclampsia originates with shallow cytotrophoblast invasion and incomplete endovascular invasion thereby inhibiting essential morphological changes of the maternal uterine vasculature from occurring. ${ }^{16,17}$ During normal pregnancy, tumor necrosis factor (TNF)- $\alpha$ promotes expression of adhesion molecules in maternal endothelial cells and activates phagocytic cells that are important mediators of morphological changes in the uterine arteries. Under normal conditions the cytotrophoblasts undergo endovascular invasion, allowing their replacement of the endothelial and muscular linings of the uterine arterioles.
As a result of this invasion, the spiral arteriolar vessel diameter increases allowing enhanced perfusion to meet the metabolic needs of the growing uteroplacental unit. By the end of the second trimester, the cytotrophoblasts line the decidua, and endothelial cells are no longer visible. However, during pre-eclampsia, cytotrophoblast invasion of the uterus is shallow, resulting in the mean arterial diameter of the first third of myometrial vessels being approximately half of that of vessels isolated from normal pregnant placentas. ${ }^{16,17}$ As a result, the spiral arteries become high resistance vessels. Furthermore, the endothelial lining of the maternal vasculature remains, allowing interactions with activated immune cells and proinflammatory cytokines to persist, leading to characteristics of a chronic inflammatory condition. ${ }^{16-19}$

Doppler velocimetric parameters have recently become established as the state-of-the-art mechanism to examine resistance index, pulsatility index, and systolic/diastolic ratio of the maternal-fetal circulation. Women developing preeclampsia have significantly altered velocimetric parameters, indicative of high vascular resistive index, compared with women with normal uterine perfusion. ${ }^{29}$ Recently, Souza and colleagues examined the effect of magnesium sulfate, a therapeutic used for prevention of seizures during eclamptic events, on parameters measured by Doppler flow velocity in the uterine, umbilical, and middle cerebral arteries in severely preeclamptic women. ${ }^{20}$ Resistance and pulsatility indices and systolic/diastolic ratios were examined in preeclamptic women before and 20 minutes after administration of magnesium sulfate. The authors found that administration of magnesium sulfate significantly decreased systolic, diastolic, and maternal blood pressure, as well as decreased resistance index in uterine arteries. ${ }^{20}$ The authors conclude that magnesium sulfate could potentially improve the resistance index within the uteroplacental circulation. Therefore, the objectives of this review are to discuss IL-6-induced pathophysiology during pregnancy, more specifically pre-eclampsia, and the potential therapeutic role for magnesium sulfate.

\section{IL-6 secretion during pathophysiological events of pregnancy}

While several groups have suggested a potential role for inflammatory cytokines in the etiology of pre-eclampsia and preterm labor, much remains unknown concerning factors stimulating the increase of these cytokines. ${ }^{18,19,21-30}$ Freeman and colleagues recently examined changes in inflammatory markers prospectively during pregnancy and the current inflammatory status of women who had a pregnancy 
complicated by pre-eclampsia 20 years previously against matched controls and found that pre-eclampsia was associated with short- and long-term changes in inflammatory status. ${ }^{23}$ Pre-eclampsia is characterized by compromised vascular remodeling, which results in decreased placental perfusion and creates an hypoxic environment for placental and fetal tissues. Under hypoxic conditions, placental explants from preeclamptic women exhibit a two-fold increase in TNF- $\alpha$ compared with explants from normal pregnant women. ${ }^{21}$ Preeclamptic women have a two-fold elevation in placental and plasma TNF- $\alpha$ protein levels. ${ }^{21}$ Circulating levels of other pro-inflammatory cytokines, such as IL-8 and IL-6, are also significantly elevated in preeclamptic women. Interestingly, circulating IL-10, an anti-inflammatory cytokine, is decreased in this same patient population. ${ }^{18}$

We now know that pre-eclampsia is associated with increased IL-6 and CD4+ T cells and Th-17 cells and decreased $\mathrm{T}$ regulatory mechanisms; however, it is unknown whether placental ischemia is a stimulus for the imbalance among Th cells..$^{33-35} \mathrm{~T}$ cells divide and secrete ILs and cytokines, such as IL-6, and assist other immune cells, such as B lymphocytes, in production of immunoglobulin. ${ }^{30,36,39}$ IL-6 is known to be a major stimulus for B-cell proliferation and serves as a regulator of many T-cell signaling processes. $^{30,36,37}$

Previous studies show that in rodents and humans, polarization and differentiation of autoimmune associated Th-17 cells from naïve Th- 0 cells is dependent upon a few cytokines, one being IL- $6 .{ }^{31-39}$ For full activation of $\mathrm{B}$ lymphocytes, interaction with activated $\mathrm{T}$ lymphocyte via the CD4 receptor and several costimulatory molecules occurs. The pathway of antibody production, referred to as the $\mathrm{T}$ cell dependent pathway, is the route for immunological memory that results in a more robust antibody response to the same antigen. This pathway is mediated through macrophages, dendritic cells, and $\mathrm{T}$ lymphocytes of the Th-1 lineage, which secrete cytokines such as TNF- $\alpha$, IL-1, IL-6, and IL-8, all of which are elevated in preeclamptic women. ${ }^{22,23,29,30,31-39}$ Therefore, elevated IL-6 could be one mechanism facilitating B lymphocyte production of AT1-AA in response to placental ischemia during pre-eclampsia.

\section{IL-6 mediates hypertension during pregnancy}

While inflammatory cytokines such as IL-6 have been reported by some laboratories to be elevated in preeclamptic women, it has been uncertain whether moderate and long-term increases in cytokines during pregnancy could result in elevations in blood pressure. We previously reported that chronic reductions in uterine perfusion pressure (RUPP) in the pregnant rat increases arterial pressure and impairs endothelial function. ${ }^{40,41}$ We have previously reported that RUPP in pregnant rats results in a chronic inflammatory response, characterized by elevated circulating TNF- $\alpha$, IL- 6 , and immune infiltrates in the placenta. ${ }^{25,26,30,35,36}$ In addition, we previously reported that a reduction in placental perfusion (RUPP) is an important stimulus for AT1-AA production in pregnant rats ${ }^{42,43}$ (Figure 1).

While AT1-AAs are elevated in response to placental ischemia, the mechanisms linking placental ischemia and AT1-AA production have yet to be fully elucidated. Our previous data show that elevated AT1-AA leads to activation of endothelin-1, oxidative stress, antiangiogenic factors, and increased sensitivity to angiotensin II (Figure 1). . $^{35,36,42,43}$ However, it is possible that the generation of AT1-AA in response to RUPP is secondary to the increased maternal inflammatory response that is associated with placental ischemia in pregnant rats. We recently reported that serum levels of IL-6 were elevated in RUPP rats, and chronic infusion of IL-6 into pregnant rats increased arterial pressure and decreased renal plasma flow and glomerular filtration rate. ${ }^{30}$ Furthermore, our recent data showed that IL-6-treated pregnant rats had significantly higher plasma renin activity

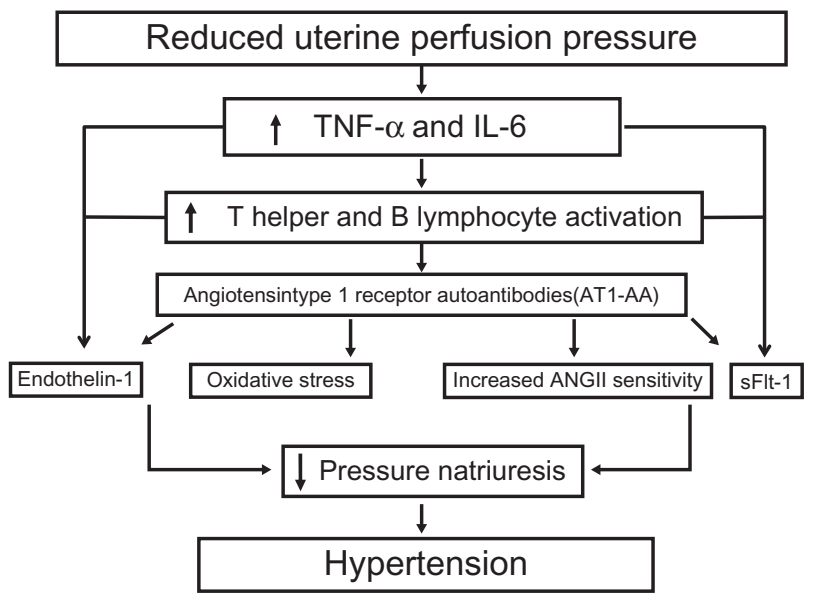

Figure I Potential role for immune activation in mediating the pathophysiology of hypertension during pre-eclampsia. Inflammatory cytokines, TNF- $\alpha$, and IL-6, stimulated in response to placental ischemia play an important role in the generation of reactive oxygen species, production of sFlt-I, and enhanced ET-I and ANGII sensitivity. One potential mechanism is stimulation of $T$ helper I and B lymphocyte maturation and secretion of ATI-AA. ATI-AA has been shown by our and other laboratories to mediate activation of ET-I, oxidative stress pathways, sFlt-I, and most recently increased sensitivity to ANGII, thereby contributing to the development of hypertension during pregnancy.

Abbreviations: ANGII, angiotensin II; ATI-AA, angiotensin II type I receptor antibodies; ET-I, endothelin-I; IL, interleukin; sFlt-I, fms-like tyrosine kinase-I; TNF, tumor necrosis factor. 
(PRA) when compared with control pregnant animals. ${ }^{30}$ Our laboratory has recently reported that in addition to increased PRA, infusion of IL-6 during pregnancy stimulates production of AT1-AA, which does not occur in virgin rats. ${ }^{43}$ Furthermore, hypertension in response to IL-6 is attenuated with an AT1 receptor antagonist, losartan, indicating the importance of IL-6 to stimulate B lymphocyte secretion of the AT1-AA as a mechanism leading to increases in blood pressure during pregnancy. ${ }^{43}$ Although this effect of IL-6 could potentially lead to enhanced vasoconstriction, reduced sodium excretory function, and hypertension via activation of the renin-angiotensin system and AT1-AAs, the quantitative importance of magnesium sulfate to reduce the in-vivo effects of IL-6 during pregnancy is unknown and remains to be an important area of investigation.

\section{The potential beneficial effects of magnesium sulfate to normalize IL-6 actions and secretion}

Over the years, many medications have been touted as both therapeutic and prophylactic agents for convulsions. Multiple studies were carried out to discern which medication was the most effective and safe in this patient population. One of the largest of these trials was the Magnesium Sulfate for Prevention of Eclampsia or Magpie Trial. ${ }^{44}$ This trial randomized women with pre-eclampsia to either magnesium sulfate or placebo for seizure prophylaxis. The results showed that treatment with magnesium sulfate lowered the number of patients who developed eclampsia by about half, without any serious harmful effects on either the mother or fetus. ${ }^{44}$ There have since been other studies which have come to the same conclusion that magnesium sulfate is the drug of choice for both the prevention and treatment of eclamptic seizures. ${ }^{45-48}$

In 2004, Holcberg et al examined the effects of magnesium sulfate on angiotensin II (ANGII)-stimulated cytokine secretion from the perfused placenta. ${ }^{49}$ The authors examined the effect of magnesium sulfate on bolus ANGII perfusion of maternal versus fetal side of placental tissue. Increasing concentrations of ANGII increased vasoconstriction of the human placental vasculature which was attenuated by addition of magnesium sulfate ( $7 \mathrm{mg} \%$; constant infusion). Subsequently, the authors measured magnesium sulfate effects on TNF- $\alpha$ and IL- 6 secretion from maternal and fetal sides of the placenta in response to bolus ANGII. The authors obtained conflicting results, giving no conclusive evidence on the effect of magnesium sulfate to reduce ANGII-induced cytokine secretion. Although these authors demonstrate a role for magnesium sulfate to abolish ANGII-induced placental vasoconstriction, the effects of magnesium sulfate to attenuate ANGII inflammation and/or AT1-AA heightened sensitivity to ANGII is unknown, thereby leaving this an important area of investigation.

In 2010, Amash et al demonstrated that preeclamptic placentas secreted significantly greater IL-6 from both maternal and fetal sides 6 hours following perfusion of control cell culture medium. ${ }^{50}$ However, addition of magnesium sulfate to the perfusion medium resulted in a tendency toward lower IL-6 secretion on the fetal side throughout the perfusion period; unfortunately this tendency did not reach statistical significance. IL-6 levels in the maternal circulation of preeclamptic placentas were significantly lower in the presence of magnesium sulfate, when compared with IL-6 levels in the maternal circulation of the preeclamptic control group, at the end of perfusion. These data support a potential anti-inflammatory role for magnesium sulfate within the ischemic placenta.

The mechanisms of action for magnesium sulfate are incompletely understood. There are many theories to explain why it seems to work. Magnesium has been shown to act as a peripheral vasodilator and thereby lower systemic blood pressure. It has also been shown to protect the blood-brain barrier and limit cerebral edema. Finally, it is thought to raise the seizure threshold through its interaction with the cell membrane. ${ }^{46-50}$ The recent work showing that magnesium sulfate may also play a role in altering the inflammatory response is exciting because it suggests that magnesium sulfate may be able to alter some of the proinflammatory mediators of endothelial dysfunction and hypertension during pre-eclampsia. ${ }^{46-51}$

In addition to pre-eclampsia, elevated IL-6 in umbilical cord blood was demonstrated to be associated with histological chorioamnionitis. ${ }^{28}$ Elevated IL-6 has been notoriously linked to preterm labor and infant neurological impairment. ${ }^{27,28}$

Studies from Tam Tam et al demonstrated that administration of magnesium sulfate pre- and post-lipopolysaccharide (LPS) treatment in a pregnant rat model of preterm labor significantly decreased maternal circulating LPS-induced TNF- $\alpha$ and IL-6 levels. ${ }^{51}$ No significant reductions in serum inflammatory concentrations were observed when magnesium sulfate was administered only before or only after LPS treatment. Furthermore, these investigators demonstrated significant decreases in TNF- $\alpha$, IL-6, and monocyte 
chemotactic protein (MCP)-1 levels in the amniotic fluid in response to antenatal magnesium sulfate therapy when administered pre- and post-LPS. In addition, maternal magnesium sulfate treatment pre- and post-LPS injection reduced fetal brain cytokine MCP-1 levels. The authors conclude that maternal administration of magnesium sulfate significantly reduces proinflammatory cytokines in maternal and fetal compartments therefore indicating that maternal magnesium sulfate administration during maternal infection could suppress damaging inflammation and improve birth outcome.

\section{Future directions and conclusions}

Previous studies demonstrated that IL- 6 is produced by placental trophoblasts and decidual cells, epithelial cells, and immune cells. Maternal infection and/or placental ischemia and reduced uterine blood flow are all pathophysiological events of pregnancy that are associated with many inflammatory cytokines, such as IL-1, IL-6, IL-8, and TNF- $\alpha$. This inflammatory response may contribute to cerebral lesions and fetal programming of disease diagnosed following birth. Magnesium sulfate is speculated to have neuroprotective effects and exhibits a decrease in cerebral inflammatory cytokines in LPS animal models of infection during pregnancy. Therefore, if magnesium sulfate alters cytokine production in infants born to infected or placental ischemic mothers, it could lend explanation to neuroprotective properties.

Although these studies have shown efficacy of magnesium sulfate to decrease inflammation during pregnancy in response to LPS, studies indicating a role for magnesium sulfate to decrease inflammation in response to placental ischemia are lacking. Furthermore, a role for magnesium sulfate to decrease immune cell activation leading to tissue infiltrates or AT1-AA in response to chronic IL-6 still needs to be demonstrated. In addition, a protective role for magnesium sulfate to blunt activation of renin-angiotensin system has yet to be illustrated. Therefore, we must conclude that although data exist supporting an anti-inflammatory role for magnesium sulfate from ex-vivo placental perfusion models and models of LPS-induced infection, appropriate studies illustrating a clinically important role for magnesium sulfate to reduce inflammation in animal models of placental ischemia are still needed, thus leaving an important preeclamptic research question unanswered.

\section{Disclosure}

The authors report no conflicts of interest in this work.

\section{References}

1. Roberts JM, Pearson G, Cutler J, Lindheimer M. Summary of the NHLBI Working Group on Research on Hypertension During Pregnancy. Hypertension. 2003;41:437-445.

2. Thadhani RI, Johnson RJ, Karumanchi SA. Hypertension during pregnancy: a disorder begging for pathophysiological support. Hypertension. 2005;46:1250-1251.

3. Roberts JM, Gammill H. Insulin resistance in pre-eclampsia. Hypertension. 2006;47:341-342.

4. Roberts JM, Gammill HS. Pre-eclampsia: recent insights. Hypertension. 2005;46:1243-1249.

5. Germain AM, Romanik MC, Guerra I, et al. Endothelial dysfunction: a link among pre-eclampsia, recurrent pregnancy loss, and future cardiovascular events? Hypertension. 2007;49:90-95.

6. Blaauw J, Graaff R, van Pampus MG, et al. Changes in endothelial function precede the clinical disease in women in whom pre-eclampsia develops in response: endothelial function and pre-eclampsia. Hypertension. 2006;47:e14-e15.

7. Khan F, Belch JJF, Macleod M, Mires G. Changes in endothelial function precede the clinical disease in women in whom pre-eclampsia develops. Hypertension. 2005;46:1123-1128.

8. Myers J, Mires G, Macleod M, Baker P. In pre-eclampsia, the circulating factors capable of altering in vitro endothelial function precede clinical disease. Hypertension. 2005;45:258-263.

9. Chambers JC, Fusi L, Malik IS, Haskard DO, De Swiet M, Kooner JS. Association of maternal endothelial dysfunction with pre-eclampsia. JAMA. 2001;285:1607-1612.

10. Granger JP, Alexander BT, Llinas MT, Bennett WA, Khalil RA. Pathophysiology of hypertension during pre-eclampsia: linking placental ischemia with endothelial dysfunction. Hypertension. 2001;38(3 Pt 2): 718-722.

11. Granger JP, Alexander BT, Bennett WA, Khalil RA. Pathophysiology of pregnancy-induced hypertension. Microcirculation. 2002;9:147-160.

12. Roberts JM, Von Versen-Hoeynck F. Maternal fetal/placental interactions and abnormal pregnancy outcomes. Hypertension. 2007;49: $15-16$.

13. Sabour S, Franx A, Rutten A, et al. High blood pressure in pregnancy and coronary calcification. Hypertension. 2007;49:813-817.

14. Mello G, Parretti E, Marozio L, et al. Thrombophilia is significantly associated with severe pre-eclampsia: results of a large-scale, casecontrolled study. Hypertension. 2005;46:1270-1274.

15. Irgens HU, Reisater L, Irgens LM, Lie RT, Roberts JM. Long term mortality of mothers and fathers after pre-eclampsia: population based cohort study Pre-eclampsia and cardiovascular disease later in life: who is at risk? BMJ. 2001;323:1213-1217.

16. Poston L. Endothelial dysfunction in pre-eclampsia. Pharmacol Rep. 2006;58 Suppl:69-74.

17. McMaster MT, Ahou Y, Fisher SJ. Abnormal placentation and the syndrome of pre-eclampsia. Semin Nephrol. 2004;24(6):540-547.

18. Sharma A, Satyam A, Sharma JB. Leptin, IL-10, and inflammatory markers (TNF-alpha, IL-6 and IL-8) in preeclamptic, normotensive pregnant and healthy non-pregnant women. Am J Reprod Immunol. 2007;58(1):21-30.

19. Girardi G, Yarilin D, Thurman JM, et al. Complement activation induces dysregulation of angiogenic factors and causes fetal rejection and growth restriction. JEM. 2006;203:2164-2175.

20. Souza AS, Amorim MM, Coutinho IC, Lima MM, Noronha NC, Figueria JN. Effect of the loading dose of $\mathrm{MgSO} 4$ on the parameters of Doppler flow velocity in the uterine, umbilical and middle cerebral arteries in severe pre-eclampsia. Hypertens Pregnancy. 2010;29:123-134.

21. Conrad KP, Benyo DF. Placental cytokines and the pathogenesis of pre-eclampsia. Am J Reprod Immunol. 1997;37:240-249.

22. Wang Y, Walsh SW. TNF alpha concentrations and mRNA expression are increased in preeclamptic placentas. J Reprod Immunol. 1996;32: $157-169$. 
23. Freeman DJ, McManus F, Brown EA, et al. Short- and long-term changes in plasma inflammatory markers associated with pre-eclampsia. Hypertension. 2004;44:708-714.

24. Armanini D, Calo LA. Aldosterone, inflammation, and pre-eclampsia. Hypertension. 2005;45:e10.

25. LaMarca BB, Bennett WA, Alexander BT, Cockrell K, Granger JP. Hypertension produced by reductions in uterine perfusion in the pregnant rat: role of tumor necrosis factor-alpha. Hypertension. 2005;46: 1022-1025.

26. LaMarca BB, Cockrell K, Sullivan E, Bennett W, Granger JP. Role of endothelin in mediating tumor necrosis factor-induced hypertension in pregnant rats. Hypertension. 2005;46:82-86.

27. Lucovnik M, Kornhauser-Cerar L, Premru-Srsen T, Gmeiner-Stopar T, Derganc M. Neutrophil defensins but not IL-6 in vaginal fluid after preterm premature rupture of membranes predict fetal/neomatal inflammation and infant neurological impairment. Acta Obstet Gynecol Scand. May 12, 2011. [Epub ahead of print].

28. Duncombe G, Veldhuizen RA, Gratton RJ, Han VK, Richardson BS. IL-6 and TNF alpha across the umbilical circulation in term pregnancies: relationship with labor events. Early Hum Dev. 2010;86:113-117.

29. Leik C, Walsh SW. Neutrophils infiltrate resistance-sized vessels of subcutaneous fat in women with pre-eclampsia. Hypertension. 2004;44:72-77.

30. Gadonski G, LaMarca BB, Sullivan E, Bennett W, Chandler D, Granger JP. Hypertension produced by reductions in uterine perfusion in the pregnant rat: role of interleukin 6. Hypertension. 2006;48: 711-716.

31. Abbus A. Cellular and Molecular Immunology. 5th ed. Philadelphia, PA: Elsevier; 2005.

32. Wulff H, Pennington M. Targeting effector memory T-cells with Kv1.3 blockers. Curr Opin Drug Discov Devel. 2007;10:438-445.

33. Rutella S. Granulocyte colony-stimulating factor for the induction of T-cell tolerance. Transplantation. 2007;84(1 Suppl):S26-S30.

34. Santner-Nanan B, Peek MJ, Khanam R, et al. Systemic increase in the ratio between Foxp3+ and IL-17-producing CD4+ T Cells in healthy pregnancy but not in pre-eclampsia. J Immunol. 2009;183: 7023-7030.

35. LaMarca B. Progress toward identifying potential markers for pre-eclampsia: role of agonistic autoantibodies to the angiotensin II type I receptor. Hypertension. 2010;55:236-237.

36. LaMarca B. The role of immune activation in contributing to vascular dysfunction and the pathophysiology of hypertension during pre-eclampsia. Minervia Ginecologica. 2010;62:105-120.

37. Panayi GS. B cells: a fundamental role in the pathogenesis of rheumatoid arthritis? Rheumatology (Oxford). 2005;44 Suppl 2:ii3-ii7.
38. Heo YJ, Joo YB, Oh HJ, et al. IL-10 suppresses Th17 cells and promotes regulatory $\mathrm{T}$ cells in the $\mathrm{CD} 4(+) \mathrm{T}$ cell population of rheumatoid arthritis patients. Immunol Lett. 2010;127:150-156.

39. Hasegawa M, Fujimoto M, Takehara K, Sato S. Pathogenesis of systemic sclerosis: altered B cell function is the key linking systemic autoimmunity and tissue fibrosis. J Dermatol Sci. 2005;39:1-7.

40. Granger JP, LaMarca BBD, Cockrell K, et al. Reduced uterine perfusion pressure (RUPP) model for studying cardiovascular-renal dysfunction in response to placental ischemia. Methods Mol Med. 2006;122: 383-392.

41. LaMarca BB, Speed J, Fournier L, Babcock SA, Berry H, Cockrell K, Granger JP. Hypertension in response to chronic reductions in uterine perfusion in pregnant rats: effect of TNF alpha blockade. Hypertension. 2008;52:1-5.

42. LaMarca BB, Wallukat G, Llinas M, Herse F, Dechend R, Granger JP. Elevated agonistic autoantibodies to the angiotensin type 1 (AT1-AA) receptor in response to placental ischemia and TNF alpha in pregnant rats. Hypertension. 2008;52:7-11.

43. Speed J, Fournier L, Cockrell K, Dechend R, Granger J, LaMarca B. IL-6 induced hypertension in pregnant rats is associated with production agonistic autoantibodies to the angiotensin II type I receptor. Presented at Experimental Biology; 2009 Apr 5; New Orleans, LA.

44. Duley L, Farrell B, Spark P, et al. Do women with pre-eclampsia, and their babies, benefit from magnesium sulfate? The Magpie Trial: a randomized placebo-controlled trial. Lancet. 2002;359:1877-1890.

45. Duley L, Henderson-Smart D. Magnesium sulphate versus phenytoin for eclampsia. Cochrane Database Syst Rev. 2003;4:CD000128.

46. Duley L, Henderson-Smart D. Magnesium sulphate versus diazepam for eclampsia. Cochrane Database Syst Rev. 2010;12:CD000127.

47. Duley L, Henderson-Smart D. Magnesium sulphate and other anticonvulsants for women with pre-eclampsia. Cochrane Database Syst Rev. 2010;11:CD000025.

48. Euser AG, Cipolla MJ. Magnesium sulfate for the treatment of eclampsia, a brief review. Stroke. 2009;40:1169-1175.

49. Holcberg G, Amash A, Sapir O, et al. Different effects of magnesium sulfate and angiotensin II on the capacity of the fetal and maternal compartments of normal human placenta to secrete TNF-alpha and IL-6. J Reprod Immunol. 2006;69:115-125.

50. Amash A, Holcberg G, Sheiner E, Huleihel M. Magnesium sulfate normalizes placental interleukin-6 secretion in pre-eclampsia. J Interferon Cytokine Res. 2010;30:683-690.

51. Tam Tam HB, Dowling O, Xue X, Lewis D, Rochelson B, Metz CN. Magnesium sulfate ameliorates maternal and fetal inflammation in a rat model of maternal infection. April 2011. Am J Obstet Gynecol. 2011; 204:364.e1-e8.

International Journal of Interferon, Cytokine and Mediator Research

Dovepress

\section{Publish your work in this journal}

The International Journal of Interferon, Cytokine and Mediator Research is an international, peer-reviewed, open-access, online journal. The focus of the journal is to publish original research, reports, editorials, reviews and commentaries on all aspects of interferon, cytokine and mediators of inflammation from labora- tory science to therapeutic indications and clinical studies. The manuscript management system is completely online and includes a very quick and fair peer-review system, which is all easy to use. Visit http://www.dovepress.com/testimonials.php to read real quotes from published authors. 